\title{
Géotechnique reviewers
}

The following is a list of referees who have reviewed papers for Géotechnique between 1 December 2013 and 30 November 2014. The Institution of Civil Engineers is very grateful for their assistance.

Professor H. Abuel-Naga

Professor M. Achmus

Professor C. Agan

Dr D. Airey

Professor E. Alonso

Mr F. Alonso-Marroquin

Dr K. Alshibli

Dr A. Al-Tabbaa

Dr G. Alvarado

Dr A. Amorosi

Professor G. Anagnostou

Professor I. Anastasopoulos

Mr K. Andersen

Dr J. Apted

Dr M. Arroyo

Professor C. Aubeny

Dr C. Augarde

Professor G. Barla

Dr D. Barreto

Dr B. Barrett

Dr B. Baudet

Mr C. Baxter

Dr K. Been

Dr G. Biscontin

Dr D. Boldini

Professor M. Bolton

Dr S. Bonelli

Professor G. Bouckovalas

Dr P. Bourne-Webb

Dr L. Boutonnier

Dr B. Bradley

Mr A. Bradshaw

Dr S. Brandenberg

Dr A. Brennan

Dr L. Bressani

Dr H. Burd

Professor J. Burland

Mr M. Burlingame

Dr S. Burlon

Professor G. Buscarnera

Dr F. Cafaro

Professor L. Callisto

Professor J. Carter

Dr F. Casini

Professor M. Cassidy

Dr A. Charles

Dr E. Chen

Professor Q. Chen

Dr H. Cheng

Dr Y. Cheng

Dr Y. P. Cheng

Dr J. Christian

Dr S. Clarke

C. Clayton

Dr P. Cleall

Dr E. Comodromos

Professor N. Consoli

Professor M. Coop

Dr D. Cortes

Dr F. Cotecchia

Dr W. Craig
Professor G. Crosta

Professor M. Cubrinovski

Dr O. Cuisinier

Professor Y. Dafalias

Dr A. Das

Dr S. Dashti

Dr J. de Bono

Professor P. de Buhan

Dr R. Dean

Dr J. Dejong

Professor P. Delage

Dr F. Delgado-Ramos

Dr G. Della Vecchia

Dr A. Diambra

Dr T. Dijkstra

Dr J. Dijkstra

Professor T. Do Anh

Dr D. Edwards

Dr G. Elia

Dr M. Elshafie

Professor B. Erich

Professor J. Evans

Professor R. Fell

Mr C. Feng

Dr G. Fenton

Dr A. Ferrari

Dr P. Ferreira

Dr F. Ferri

Dr J. Fonseca

Professor A. Gajo

Professor D. Gallipoli

Dr A. Gasparre

Professor C. Gaudin

Dr K. Gavin

Professor G. Gazetas

Dr F. Gelagoti

Dr K. Georgiadis

Dr V. Georgiannou

Dr P. Gerard

Dr N. Gerolymos

Dr S. Ghabezloo

Mr M. Goudarzy

Mr J. Grabe

Dr V. Greco

Professor R. Green

Dr P. Gueguen

Dr S. Haigh

Dr D. Hanaor

Dr M. Harbottle

Mr D. Hartwell

Professor Y. Hashash

Professor I. Herle

Dr C. Heron

Professor P.-Y. Hicher

Professor M. Hicks

Dr D. Hight

Professor A. Holeyman

Dr M. Hossain

Professor G. Houlsby

Professor H. Huang

Dr D. Hughes
Dr P. Hughes

Dr A. Hunt

Dr N. Hytiris

Dr E. Ibraim

Professor B. Indraratna

Dr T. Ishikawa

Dr M. Iskander

Professor S. Jacobsz

Professor M. Jefferies

Dr R. Jibson

Professor C. Jommi

Dr A. Jotisankasa

Dr V. Jovicic

Dr W. Kanning

Dr M. Karstunen

Professor M. Kavvadas

Professor N. Khalili

Dr J. Kinuthia

Dr M. Kitazume

Dr T. Kiyota

Professor A. Klar

Dr J. Knappett

Dr J. Kodikara

Professor D. Kolymbas

Dr A. Kottke

Dr T. Koumoto

Dr G. Kouretzis

Dr R. Kourkoulis

Dr N. Kovacevic

Dr J. Krahn

Mr G. Kruse

Dr M. Kuhn

Professor B. Kutter

Mr A. Kwok

Mr F. Kwok

Professor P. Lade

Professor R. Lagioia

Dr C. Lam

Professor R. Lancellotta

Dr L. Le Pen

Professor J.-S. Lee

Professor B. Lehane

Dr C. Leo

Dr E. Leong

Professor S. Leroueil

Professor C. Leung

Dr L. Li

Professor H. Ling

Professor J.-C. Liu

Mr D. Lo Presti

Dr A. Locat

Dr M. Long

Dr D. Loukidis

Dr J. Love

Dr J. Lovisa

Professor N. Lu

Mr Y. Luo

Professor A. Lutenegger

Dr G. Madabhushi

Dr G. Marketos

Dr A. Marshall
Dr D. Masin

$\mathrm{Mr}$ T. Matsumoto

Dr J. McCartney

Dr J. McDougall

Dr Z. Medina-Cetina

Mr X. Mei Guo

Mr A. Merritt

Professor G. Mesri

Professor R. Michalowski

Dr B. Montoya

Professor D. Muir Wood

Dr G. Musso

Dr S. Najjar

Professor Y. Nakata

Dr D. Nash

Dr V. Navarro

Dr T. Newson

Professor C. Ng

Professor T.-T. Ng

Dr H. Nguyen

Professor F. Nicot

Dr S. Nishimura

Professor S. Nordal

Dr J. Oliveira

Dr T. Orr

Dr A. Osman

Dr C. O’Sullivan

Mr J. Otani

Mr A. Pak

Professor K. Panagiotis

Professor A. Papadimitriou

Mr I. Papaioannou

Professor E. Pasternak

Professor J. Pastor

Professor M. Pastor

Professor M. Pender

Dr J.-M. Pereira

Dr J. Pineda

Dr M. Pirone

Dr F. Pisano

Mr D. Pitilakis

Professor K. Pitilakis

Professor D. Potts

Professor W. Powrie

Dr M. Preene

Dr J. Priest

Dr T. Pucker

Professor A. Puppala

Professor S. Rampello

Professor M. Randolph

Professor E. Rathje

Dr S. Rees

Professor D. Richards

Dr T. Roberts

Dr P. Robertson

Dr P. Rognon

Dr E. Romero-Morales

Mr H. Roscoe

Dr J.-N. Roux

Dr C. Rujikiatkamjorn

Dr A. Russell 
Professor R. Salgado

Dr F. Santucci de Magistris

Dr S. Sarma

Professor T. Schanz

Dr J. Schneider

Dr D. Schreiner

Professor H. Schweiger

Dr S. Sepulveda

Dr E. Seyedi Hosseininia

Dr M. Sharp

Professor D. Sheng

Professor S. Shibuya

Dr D. Shuttle

Mr F. Silvestri

Dr W. Sim

Professor P. Simms

Dr B. Simpson

Dr V. Sivakumar

Dr K. Skoglund

Dr C. Smith

Professor K. Soga

Dr W. Solowski
Dr C. Song

Professor A.-H. Soubra Professor S. Springman

Dr S. Stallebrass

Dr J. Standing

Mr A. Stavrou

Dr I. Stefanou

Dr D. Sterpi

Dr D. Stewart

Dr K. Stone

Professor J. Sulem

Dr D. Taborda

Dr H. Taiebat

Professor W. Take

Mr M. Talesnick

Professor C. Tamagnini

Dr A. Tang

Professor A. Tarantino

Professor F. Tatsuoka

Professor L. Tham

Dr M. Thomas

Mr N. Thusyanthan
Professor A. Tordesillas Professor I. Towhata Dr S. Tripathy Professor A. Troncone Dr A. Tsiampousi Dr S. Utili

Professor L. Vallejo

Dr L. Van Paassen

Dr P. Vardanega

Dr P. Vardon

Dr R. Vassallo

Dr J. Vaunat

Professor A. Verruijt

Dr M. Veveakis

Professor G. Viggiani

Dr M. Villar

Dr J. S. Vinod

Dr D. Wang

Dr S. Wersching

Dr J. West

Dr D. White

Professor A. Whittle
Dr T. Wichtmann

Mr Z. Wieckowski

Dr L. Wong

Professor T.-F. Wong

Professor P. Woodward

Dr L. Wotherspoon

Dr X. Xu

Dr J. Yang

Professor N. Yasufuku

Dr J. Yi

Dr Q. Yue

Mr F. Zabala

Dr V. Zania

Professor L. Zdravkovic

Professor X. Zeng

Dr A. Zervos

Mr L. Zhan

Dr R. Zhang

Dr C. Zhao

Professor J. Zhao 